\title{
Estudo ecológico sobre o desenvolvimento da saúde no Brasil
}

\author{
Ecological study on the development of health in Brazil
}

Maria Meimei Brevidelli ${ }^{1}$

Fernando Celso Garcia de Freitas ${ }^{2}$

${ }^{1}$ Universidade Paulista -

UNIP Indianópolis. R. Dr

Bacelar 1212, Indianópolis.

04026-002 São Paulo SP.

meimei@usp.br

${ }^{2}$ Confederação Nacional de

Serviços

\begin{abstract}
The scope of this paper was to assess the health level in Brazilian states using the Health Development Index (HDI). The HDI consisted of the following dimensions: (1) Health resources: Availability and quality of health resources; (2) Primary Healthcare coverage and sanitation; (3) Effectiveness of health policies. Each dimension was composed of a set of indicators obtained from national databases. In 2005, Brazil had an intermediate level of development of health, having progressed from a low level in 1999. Most states had medium and low development, with deficits in resources and coverage. The dimension of effectiveness was highly developed nationwide. With the construction of a synthetic indicator (HDI) it was possible to detect that in most of the country there are severe deficiencies in the availability and quality of health resources. These results can help health managers to tackle the challenge of making public health universal.

Key words Health indicators, Health statistics, Health inequalities, Health management, Socioeconomic factors, Ecological studies
\end{abstract}

Resumo Avaliar o nível de saúde das Unidades da Federação (UF) do Brasil, por meio de um indicador sintético - Indice de Desenvolvimento da Saúde (IDS). O IDS foi composto pelas dimensões: (1) Recursos de saúde: disponibilidade e qualidade dos recursos de saúde; (2) Cobertura por atenção básica e saneamento; (3) Eficácia das políticas de saúde. Cada dimensão englobou um conjunto de indicadores obtidos em bases de dados nacionais. Em 2005, o Brasil apresentava nível intermediário de desenvolvimento da saúde, tendo progredido de patamares inferiores, desde 1999. A maioria das UF apresentava déficits em "recursos" e "cobertura". A dimensão da "eficácia" mostrou-se altamente desenvolvida em todo Brasil. Conclusões: A construção do indicador sintético IDS possibilitou constatar deficiências e disparidades significativas na disponibilidade e qualidade dos recursos de saúde, em grande parte do território nacional. Esses resultados podem auxiliar gestores de saúde no desafio de tornar a saúde pública universal.

Palavras-chave Indicadores de saúde, Estatísticas de saúde, Desigualdades de saúde, Gestão em saúde, Fatores socioeconômicos, Estudos ecológicos 


\section{Introdução}

As primeiras metodologias de mensuração do estado de saúde da população estavam integralmente baseadas em indicadores de mortalidade e de morbidade ${ }^{1}$. Em geral, atribuía-se maior nível de saúde às sociedades com menor número de doenças e mortes. A taxa de mortalidade infantil é um exemplo clássico, pelo qual se atribui melhores condições de saúde a populações com menores taxas de mortalidade.

Tendo em vista que a saúde é um estado dinâmico dependente da integração de fatores comportamentais, ambientais e sociais, mais recentemente a mensuração desse estado passou a incorporar indicadores sobre condições de vida, acesso a serviços de saúde e qualidade da atenção primária. No Brasil, o grupo de Indicadores e Dados Básicos $(I D B)^{2}$ reúne um vasto conjunto de indicadores demográficos, socioeconômicos, de morbi-mortalidade e fatores de risco, além de estatísticas sobre recursos e cobertura de serviços. Esses indicadores englobam diversos aspectos determinantes do estado de saúde do país e de diferentes grupos populacionais (mulheres, crianças, recém-nascidos e idosos, dentre outros). No entanto, os indicadores de saúde no Brasil são descritos de forma separada e disponibilizados de maneira dissociada.

De outro lado, há uma crescente preocupação de integrar diversas estatísticas de saúde de maneira a expressar o estado de saúde de uma população pelo seu nível de qualidade, cuja evolução no tempo possa ser visualizada e comparada. Como exemplo dessa perspectiva há os indicadores de saúde do Programa "Healthy People", do Departamento de Saúde dos Estados Unidos, cujos objetivos principais são (i) auxiliar indivíduos a aumentar a longevidade e a qualidade de vida e (ii) eliminar as disparidades dos níveis de saúde entre os segmentos da população ${ }^{3}$.

O Programa "Healthy People 2020" representa a quarta geração dessa iniciativa, nas quais os níveis de saúde são mensurados sob quatro perspectivas: (1) status da saúde em geral; (2) qualidade de vida relacionada à saúde e bem-estar; (3) determinantes sociais da saúde; e (4) disparidades e desigualdades. Para cada aspecto foram determinadas metas específicas a serem alcançadas para os próximos 10 anos, fundamentadas em um abrangente conjunto de indicadores de saúde, o que permite monitorar o progresso do nível de saúde da população americana ${ }^{4}$.

Tendo em vista a necessidade de integrar aspectos quantitativos a qualitativos na mensura- ção do nível de saúde de uma população, o presente artigo propõe um índice sintético que explora relações de complementaridade entre os Indicadores Básicos de Saúde. Esse índice, chamado de Índice de Desenvolvimento da Saúde (IDS) é então empregado para analisar o estado de saúde no Brasil e em suas unidades federativas.

\section{Métodos}

O presente artigo traz um estudo ecológico no qual as unidades de análise são os 26 Estados brasileiros e o Distrito federal. Nessas unidades foram avaliados os níveis de saúde por meio do Índice de Desenvolvimento da Saúde (IDS). Essa abordagem se aproxima metodologicamente da iniciativa de se medir o desenvolvimento populacional por meio do Índice de Desenvolvimento Humano (IDH $)^{5}$, um indicador sintético que integra a avaliação de aspectos quantitativos e qualitativos. Nesse índice, o desenvolvimento é medido por meio das comparações de PIB per capi$t a$, longevidade e educação de diferentes nações. Sua finalidade é aferir o desenvolvimento de uma população, considerando não apenas a dimensão econômica, mas também características sociais, culturais e políticas que influenciam a qualidade da vida humana ${ }^{6}$.

Seguindo essa visão, o IDS foi composto pela integração de diferentes dimensões que refletem o desenvolvimento da saúde. O presente estudo propõe que o grau de desenvolvimento da saúde de uma unidade regional espelhe a disponibilidade e a qualidade de recursos físicos e humanos, assim como a eficácia das políticas públicas, que permitem aumentar a longevidade e a qualidade de vida de sua população. Deste modo, as dimensões do IDS são: (1) a disponibilidade e a qualidade dos recursos de saúde; (2) a cobertura da atenção básica e de saneamento; (3) a eficácia de políticas públicas de saúde na redução da morbidade e mortalidade.

\section{Dimensões e componentes do IDS}

Para cada dimensão do IDS foi composto um índice formado pela associação de vários indicadores obtidos no banco de dados do Departamento de Informação e Informática do SUS (Datasus). A escolha dos indicadores sociais e de saúde de cada dimensão foi orientada pela compreensão de que estes representam recursos básicos, condições de vida prioritárias e políticas públicas que têm impacto no estado de saúde da popula- 
ção brasileira. Ainda que limitados, esses indicadores representam os princípios básicos do SUS de universalidade, integralidade e equidade. A concepção e a composição do indicador sintético foi julgada por dois especialistas, um na área de econometria e outro em epidemiologia, para verificar a capacidade dos indicadores representarem adequadamente as dimensões teóricas correlacionadas. Após essa análise, alguns indicadores foram revisados e substituídos. A Figura 1 apresenta a versão final das dimensões e dos componentes do IDS, elaborado pelos autores. A seguir descrevemos a composição de cada índice.

\section{Índice da dimensão "Disponibilidade e qualidade dos recursos de saúde"}

Nesse índice, também chamado de indice de recursos, foram associados dois componentes: quantidade e qualidade dos recursos de saúde. A quantidade de recursos de saúde expressa disponibilidade relativa, ou seja, o número de recursos de saúde em relação à população residente em uma região. Os recursos quantificados foram: (a) número de unidades ambulatoriais/mil habitantes; (b) número de leitos hospitalares/mil habitantes; (c) número de profissionais de saúde/mil habitantes. A qualidade dos recursos de saúde procura criar um indicador que ajuste a quantidade de recursos permitindo a agregação de serviços de distintas qualidades. O indicador baseia-se: (i) na proporção de procedimentos especializados, de média e alta complexidade, em relação ao total de procedimentos realizados; (ii) nos dias de internação por dias de leito ocupados; (iii) número de profissionais de saúde com nível superior e técnico.

A integração dos dois componentes partiu das seguintes premissas. Primeiro, acredita-se que a proporção de procedimentos especializados e de média e alta complexidade representa uma assistência de saúde mais complexa oferecida nas unidades ambulatoriais. Depois, entende-se que o maior tempo de internação por dias de leito ocupado é reflexo da gravidade do paciente e da complexidade da assistência de saúde prestada. Por fim, parte-se do princípio que o maior grau de instrução dos profissionais de saúde significa um maior nível de conhecimentos científicos e de competências técnicas. Assim, as quantidades ajustadas às qualidades expressariam melhor a disponibilidade efetiva de recursos.

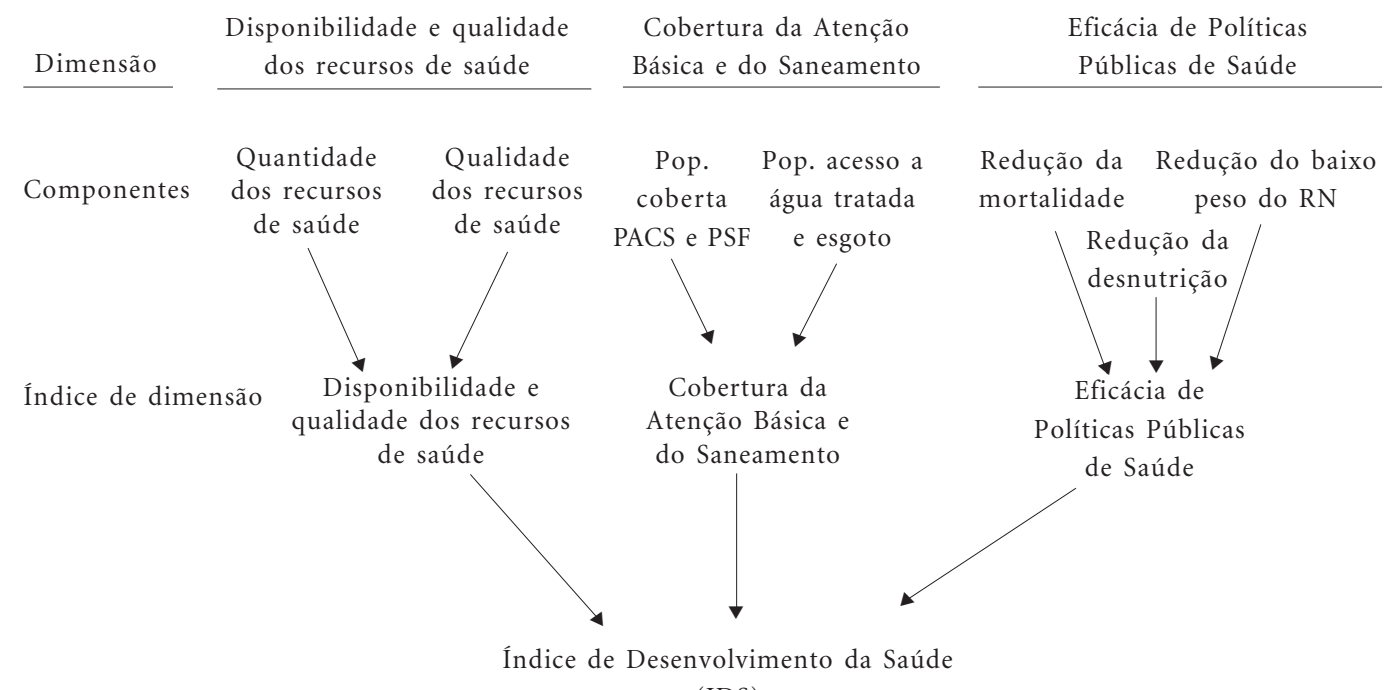

(IDS)

Figura 1. Esquema das dimensões e componentes do IDS. 
Índice da dimensão "Cobertura por atenção básica e saneamento"

No índice "Cobertura por atenção básica e saneamento", ou simplesmente indice de cobertura, foram associados os seguintes indicadores: (a) a proporção da população residente coberta por programas de atenção básica - Programa de Agentes Comunitários de Saúde (PACS) e Programa de Saúde da Família (PSF); e (b) a proporção da população residente com acesso à água tratada e ao esgotamento sanitário. Os programas de saúde selecionados representam os principais pilares do Governo Federal para reestruturação do modelo de atenção em saúde públi$\mathrm{Ca}^{7}$. Portanto, o indicador representa o acesso da população à rede de atenção à saúde e aos serviços básicos de saúde pública (água e esgoto).

\section{Índice da dimensão "eficácia de políticas públicas de saúde na redução da morbidade e mortalidade"}

Esse índice, ou simplesmente indice de eficácia, é formado por três componentes. A primeira, chamada de eficácia na redução da mortalida$d e$, associou a taxa de mortalidade infantil e a taxa de mortalidade proporcional por doenças infecto-parasitárias. A segunda componente, denominada eficácia na redução da desnutrição, empregou a proporção de crianças com menos de um ano de idade que são desnutridas. A terceira, eficácia na redução do baixo peso ao nascer, avaliou a proporção de recém-nascidos (RN) com baixo peso. Essa associação de indicadores de mortalidade e morbidade infantis buscou avaliar o impacto positivo de políticas públicas sociais, na medida em que a redução desses indicadores representa melhores condições de vida, com aumento da longevidade da população e interações positivas com a educação. A mortalidade por doença infecto-parasitária é uma das cinco principais causas de óbitos no Brasil. Portanto, esse indicador também é um bom parâmetro da eficácia dos investimentos públicos para a melhoria da qualidade de saúde.

\section{Bases de Dados e Cálculo do IDS}

Os indicadores primários - as estatísticas de cada dimensão - foram obtidos nas bases de dados do Datasus ${ }^{8}$ e na Pesquisa Nacional por Amostra de Domicílio (PNAD) do IBGE? . São médias anuais centradas em 1 de julho, obtidas para encontrar relativos com a população, ou são valores acumulados ao longo do ano. As estatísticas da PNAD referem-se a $1^{\circ}$ de setembro e, nesse caso, os relativos com a população também tem essa data de referência. "Os dados sobre despesas com saúde e saneamento foram obtidos na Secretaria do Tesouro Nacional ${ }^{10}$. Foi feito o cálculo do IDS e suas componentes para cada unidade da Federação entre 1999 e 2005. O último ano foi escolhido como referência para a análise comparativa do IDS nas diferentes unidades da Federação e sua relação com outros indicadores socioeconômicos.

O IDS é a média aritmética dos índices de recursos, de cobertura e de eficácia. Cada uma de suas componentes é padronizada em um intervalo de valores entre 0 (zero) a 1 (um). O IDS de cada unidade, bem como os índices de suas dimensões, foi classificado de acordo com o seguinte critério, para facilitar a análise: (a) Se IDS $\geq 0,750$, considera-se que o nível de desenvolvimento da saúde é alto; (b) Se 0,700 $<$ IDS $<0,750$, considera-se que o nível é intermediário; (c) Se IDS $\leq 0,700$ considera-se que o nível de desenvolvimento da saúde é baixo.

\section{Resultados}

A Figura 2 apresenta a evolução do IDS do Brasil no período entre 1999 e 2005. Entre as dimensões do IDS, percebe-se que a de eficácia é que tem valores relativamente mais elevados. Apesar disso, no período em questão o crescimento desse indicador foi pequeno, de $3 \%$.

Por outro lado, vê-se um avanço expressivo do índice de cobertura: crescimento de $27,0 \%$. De forma semelhante, observa-se um crescimento elevado, de 10\%, no indice de recursos.

O IDS do Brasil registrou um crescimento significativo no período (11\%), passando de 0,671 em 1999 para 0,746 em 2005. Isso provocou a mudança, na média nacional, do nível baixo para o médio de desenvolvimento da saúde nesses anos.

A Tabela 1 apresenta o IDS e os índices das três dimensões para as unidades da Federação em 2005. Observando as médias dos índices das três dimensões, logo se identifica que o indice de recursos foi o que obteve a média mais baixa $(0,593)$ e o que apresentou maior variabilidade entre o valor mínimo (0,471 no Maranhão) e o valor máximo $(0,805$ no Distrito Federal), revelando uma enorme heterogeneidade da quantidade e qualidade dos recursos de saúde no país.

Fica evidente também que apenas Rio de Janeiro, São Paulo e o Distrito Federal, possuíam infraestrutura relativamente mais desenvolvida 


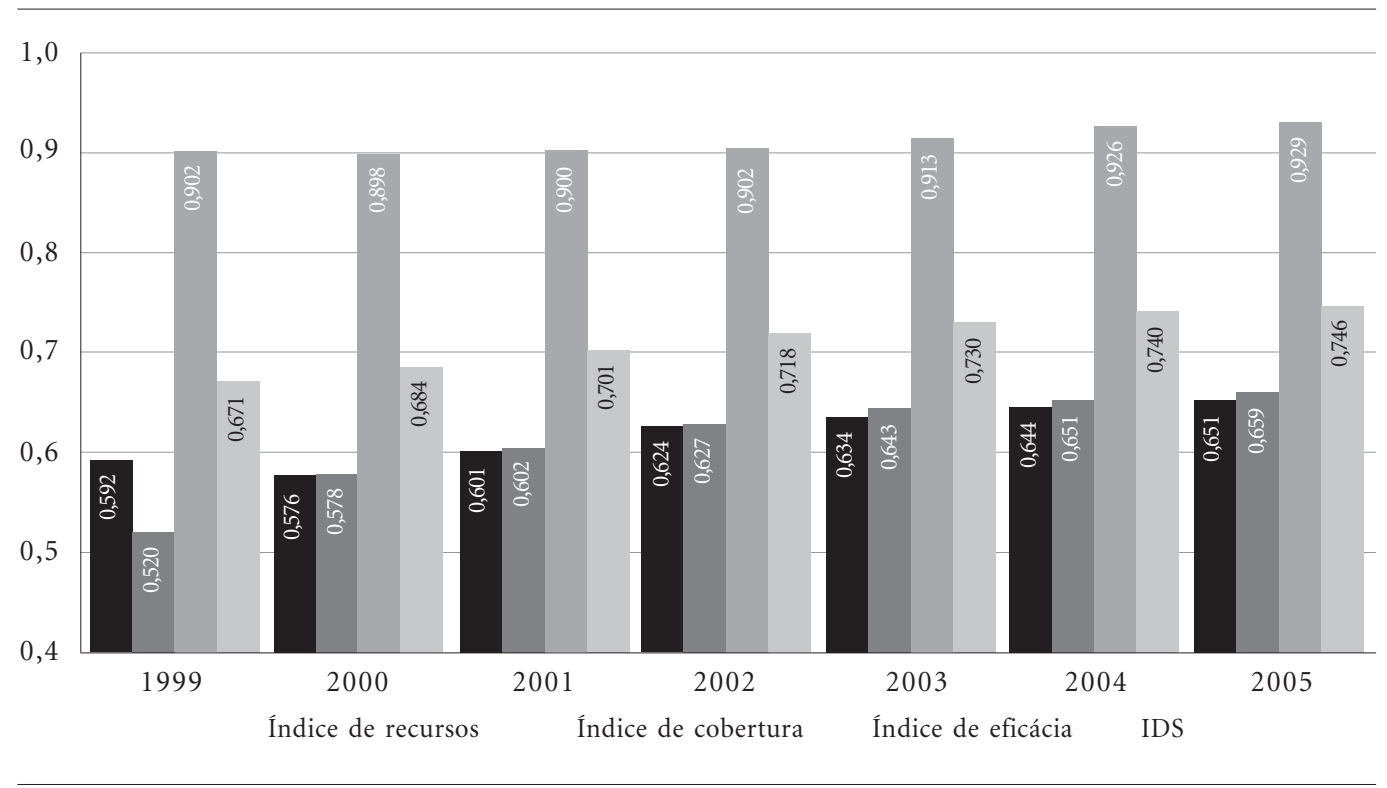

Figura 2. Índice de desenvolvimento da saúde e índices de recurso, cobertura e eficácia, Brasil, 1999-2005.

Tabela 1. Índice de Desenvolvimento da Saúde e suas dimensões, Unidades da Federação, 2005.

\begin{tabular}{|c|c|c|c|c|}
\hline Unidades da Federação & Índice de recursos & Índice de Cobertura & Índice de Eficácia & IDS \\
\hline Rondônia & 0,526 & 0,515 & 0,947 & 0,663 \\
\hline Acre & 0,611 & 0,485 & 0,899 & 0,665 \\
\hline Amazonas & 0,504 & 0,504 & 0,931 & 0,646 \\
\hline Roraima & 0,539 & 0,521 & 0,942 & 0,668 \\
\hline Pará & 0,484 & 0,476 & 0,923 & 0,627 \\
\hline Amapá & 0,578 & 0,591 & 0,943 & 0,704 \\
\hline Tocantins & 0,586 & 0,583 & 0,917 & 0,695 \\
\hline Maranhão & 0,471 & 0,497 & 0,881 & 0,617 \\
\hline Piauí & 0,576 & 0,541 & 0,891 & 0,669 \\
\hline Ceará & 0,573 & 0,597 & 0,915 & 0,695 \\
\hline Rio Grande do Norte & 0,599 & 0,622 & 0,921 & 0,714 \\
\hline Paraíba & 0,596 & 0,683 & 0,899 & 0,726 \\
\hline Pernambuco & 0,609 & 0,657 & 0,904 & 0,724 \\
\hline Alagoas & 0,510 & 0,521 & 0,880 & 0,637 \\
\hline Sergipe & 0,507 & 0,774 & 0,903 & 0,728 \\
\hline Bahia & 0,541 & 0,620 & 0,904 & 0,689 \\
\hline Minas Gerais & 0,626 & 0,758 & 0,914 & 0,766 \\
\hline Espírito Santo & 0,635 & 0,753 & 0,949 & 0,779 \\
\hline Rio de Janeiro & 0,720 & 0,684 & 0,946 & 0,783 \\
\hline São Paulo & 0,712 & 0,717 & 0,951 & 0,793 \\
\hline Paraná & 0,654 & 0,701 & 0,943 & 0,766 \\
\hline Santa Catarina & 0,665 & 0,737 & 0,945 & 0,783 \\
\hline Rio Grande do Sul & 0,690 & 0,621 & 0,949 & 0,753 \\
\hline Mato Grosso do Sul & 0,627 & 0,609 & 0,940 & 0,725 \\
\hline Mato Grosso & 0,570 & 0,589 & 0,942 & 0,700 \\
\hline Goiás & 0,594 & 0,613 & 0,934 & 0,714 \\
\hline Distrito Federal & 0,805 & 0,667 & 0,961 & 0,811 \\
\hline Brasil $^{*}$ & 0,651 & 0,659 & 0,929 & 0,746 \\
\hline Média simples & 0,593 & 0,612 & 0,922 & 0,709 \\
\hline Mínimo & 0,471 & 0,476 & 0,880 & 0,617 \\
\hline Máximo & 0,805 & 0,774 & 0,961 & 0,811 \\
\hline
\end{tabular}

NOTA: $\left({ }^{*}\right)$ os dados do Brasil são médias ponderadas pela população. 
em recursos de saúde. Em 24 estados o índice de recursos foi inferior a 0,700 , indicando que, em grande parte do território nacional, os recursos básicos de saúde eram escassos e pouco qualificados. Os valores menores foram encontrados em Maranhão (0,471), Pará $(0,484)$, Amazonas $(0,504)$, Sergipe $(0,507)$ e Alagoas $(0,510)$.

Com relação ao indice de cobertura, apenas três unidades da Federação apresentaram altos níveis: Sergipe, Minas Gerais e Espírito Santo $(0,774,0,758,0,753$, respectivamente). Além disso, Santa Catarina, São Paulo e Paraná apresentaram valores intermediários. Porém, novamente, a maioria das regiões do país tem baixo nível de desenvolvimento, incluindo o Rio de Janeiro $(0,684)$ e o Distrito Federal $(0,667)$ que, como visto anteriormente, possuíam infraestrutura de recursos de saúde mais desenvolvida. Novamente, os índices mais baixos estão nos estados das regiões Norte e Nordeste: Pará (0,476), Acre (0,485), Amazonas (0,504), Rondônia (0,515), Roraima $(0,521)$ e Maranhão $(0,497)$. Por esses motivos, $o$ indice de cobertura revelou um déficit considerável em condições básicas de vida e no acesso a programas de atenção básica.

$\mathrm{O}$ indicador de eficácia apresentou valores relativamente mais altos e também maior homogeneidade (de 0,961 no Distrito Federal a 0,880 em Alagoas). Apesar de relativamente maiores, devese observar a existência de diferenças regionais expressivas. Nos estados do Acre, Paraíba, Piauí e Alagoas, os níveis de eficácia ficam entre 0,880 e 0,899 , valores pequenos se comparados aos do Distrito Federal, de São Paulo, do Espírito Santo ou do Rio Grande do Sul (todos entre 0,961e 0,949).

Em 2005, apenas oito unidades apresentaram nível de desenvolvimento da saúde alto (superior a 0,750$)$. São eles: Distrito Federal $(0,811)$, São Paulo (0,793), Rio de Janeiro (0,783), Santa Catarina $(0,783)$, Espírito Santo $(0,779)$, Paraná $(0,766)$, Minas Gerais $(0,766)$ e Rio Grande do Sul $(0,753)$. Em geral, essas unidades se diferenciaram pelos valores mais elevados em todas as dimensões. São Paulo foi o único estado em que os índices de recursos, de cobertura e de eficácia estavam entre os cinco mais altos de cada dimensão, o que o distinguiu como o estado relativamente mais equilibrado em relação às dimensões do IDS. Por outro lado, o Distrito Federal que apresentou o IDS mais elevado, destacou-se com os melhores índices de recursos e eficácia, mas uma deficiência na cobertura.

Um grupo de sete estados apresentou níveis intermediários de desenvolvimento da saúde: Sergipe, Paraíba, Mato Grosso do Sul, Pernam- buco, Rio Grande do Norte, Goiás e Amapá. Nesse grupo, percebem-se alguns contrastes. Apesar de Sergipe ter apresentado a melhor cobertura, possuía grandes deficiências em recursos e em eficácia. O mesmo ocorreu na Paraíba. Por outro lado, no Amapá a dimensão da eficácia estava consideravelmente mais desenvolvida do que a dos recursos e cobertura.

No outro extremo, estavam os estados que apresentaram baixo nível de desenvolvimento da saúde: Mato Grosso, Tocantins, Ceará, Bahia, Piauí, Roraima, Acre, Rondônia, Amazonas, Alagoas, Pará e Maranhão. Nesses estados, os índices de recursos e cobertura estão, em geral, entre os mais baixos (inferiores a 0,600 ). Chama a atenção o caso do estado de Rondônia que, com um dos cinco IDS mais baixos, apresentou um dos cinco indices de eficácia mais elevados. Este fato indica que, nesse estado, o baixo nível de desenvolvimento da saúde foi determinado pelas deficiências nos recursos e cobertura.

A Tabela 2 apresenta as despesas per capita com saúde pública e saneamento e a participação delas nas despesas públicas totais, nos anos de 2000 e 2005. Em 2005, despendia-se cerca de R\$ 353,58 por habitante em saúde pública e saneamento. Nesse ano, essas despesas correspondiam a 16\% dos gastos correntes totais das três esferas de governo no país. Pode-se observar que, no período, houve um crescimento anual de $19,8 \%$ nesse gasto por habitante, pelo o qual o Brasil despedia cerca de $\mathrm{R} \$ 353,58$ per capita com saúde e saneamento em 2005

Para analisar se existe relação entre a despesa em saúde e o IDS, tomaram-se como exemplo dois grupos opostos: os três estados com os maiores IDS (Distrito Federal, São Paulo e Rio de Janeiro) e os três estados com os menores índices (Maranhão, Pará e Alagoas).

Inicialmente nota-se que nos dois grupos o crescimento anual do gasto foi semelhante - aproximadamente $18 \%$ ao ano entre 2000 e 2005 . No entanto, os estados do $2^{\circ}$ grupo partiram em 2000 de patamares mais baixos de gasto do que o $1^{\circ}$ grupo; por exemplo, naquele ano, Maranhão gastava R\$ 72,88 e São Paulo, R\$233,21. Consequentemente, em 2005, apesar do crescimento anual ter sido semelhante nos dois grupos, o $1^{\circ}$ grupo gastava aproximadamente $\mathrm{R} \$ 450,00$ (cada estado), enquanto o $2^{\circ}$ grupo gastava entre $\mathrm{R} \$$ 164,00 e R\$ 340,00. Essa comparação sugere a existência de uma relação positiva entre investimento em saúde/saneamento e nível do IDS.

Essa percepção é reforçada pela associação linear ilustrada pela Figura 3. Nela, observa-se 
Tabela 2. Despesas per capita em saúde e saneamento, 2000 e 2005.

\begin{tabular}{|c|c|c|c|c|c|c|}
\hline \multirow{2}{*}{ Unidade da Federação } & \multicolumn{3}{|c|}{ R\$ por habitante } & \multicolumn{3}{|c|}{ Participação nas despesas totais } \\
\hline & 2000 & 2005 & Variação anual & 2000 & 2005 & Variação anual \\
\hline Rondônia & 114,14 & 315,29 & $28,9 \%$ & $9,9 \%$ & $14,5 \%$ & $10,2 \%$ \\
\hline Acre & 349,87 & 471,07 & $7,7 \%$ & $19,4 \%$ & $15,6 \%$ & $-5,3 \%$ \\
\hline Amazonas & 91,76 & 455,79 & $49,3 \%$ & $8,1 \%$ & $21,0 \%$ & $26,8 \%$ \\
\hline Roraima & 262,21 & 492,23 & $17,1 \%$ & $11,1 \%$ & $14,2 \%$ & $6,3 \%$ \\
\hline Pará & 114,75 & 227,68 & $18,7 \%$ & $16,0 \%$ & $19,1 \%$ & $4,6 \%$ \\
\hline Amapá & 143,54 & 407,72 & $29,8 \%$ & $10,0 \%$ & $15,1 \%$ & $10,9 \%$ \\
\hline Tocantins & 156,73 & 385,44 & $25,2 \%$ & $10,6 \%$ & $15,7 \%$ & $10,2 \%$ \\
\hline Maranhão & 72,88 & 164,00 & $22,5 \%$ & $11,7 \%$ & $16,5 \%$ & $9,0 \%$ \\
\hline Piauí & 119,81 & 292,39 & $25,0 \%$ & $16,6 \%$ & $20,9 \%$ & $5,9 \%$ \\
\hline Ceará & 121,13 & 253,24 & $20,2 \%$ & $14,2 \%$ & $17,8 \%$ & $5,8 \%$ \\
\hline Rio Grande do Norte & 193,17 & 313,03 & $12,8 \%$ & $18,1 \%$ & $16,8 \%$ & $-1,9 \%$ \\
\hline Paraíba & 113,07 & 270,91 & $24,4 \%$ & $12,7 \%$ & $18,1 \%$ & $9,3 \%$ \\
\hline Pernambuco & 70,10 & 282,71 & $41,7 \%$ & $7,8 \%$ & $16,9 \%$ & $21,2 \%$ \\
\hline Alagoas & 170,18 & 338,53 & $18,8 \%$ & $19,8 \%$ & $20,8 \%$ & $1,2 \%$ \\
\hline Sergipe & 129,17 & 350,06 & $28,3 \%$ & $11,3 \%$ & $17,2 \%$ & $11,0 \%$ \\
\hline Bahia & 112,03 & 257,40 & $23,1 \%$ & $13,4 \%$ & $17,9 \%$ & $7,5 \%$ \\
\hline Minas Gerais & 181,54 & 325,45 & $15,7 \%$ & $14,1 \%$ & $16,3 \%$ & $3,8 \%$ \\
\hline Espírito Santo & 160,90 & 329,77 & $19,7 \%$ & $10,1 \%$ & $12,3 \%$ & $5,1 \%$ \\
\hline Rio de Janeiro & 233,33 & 449,97 & $17,8 \%$ & $12,3 \%$ & $15,8 \%$ & $6,4 \%$ \\
\hline São Paulo & 233,21 & 453,25 & $18,1 \%$ & $12,9 \%$ & $15,8 \%$ & $5,2 \%$ \\
\hline Paraná & 157,94 & 312,37 & $18,6 \%$ & $9,9 \%$ & $14,8 \%$ & $10,4 \%$ \\
\hline Santa Catarina & 175,89 & 372,91 & $20,7 \%$ & $11,3 \%$ & $15,3 \%$ & $7,8 \%$ \\
\hline Rio Grande do Sul & 184,44 & 358,28 & $18,1 \%$ & $12,0 \%$ & $14,7 \%$ & $5,2 \%$ \\
\hline Mato Grosso do Sul & 135,61 & 380,78 & $29,4 \%$ & $9,3 \%$ & $14,3 \%$ & $11,1 \%$ \\
\hline Mato Grosso & 159,45 & 384,65 & $24,6 \%$ & $10,8 \%$ & $14,5 \%$ & $7,8 \%$ \\
\hline Goiás & 126,11 & 350,37 & $29,1 \%$ & $11,9 \%$ & $17,2 \%$ & $9,7 \%$ \\
\hline Distrito Federal & 415,60 & 448,96 & $1,9 \%$ & $14,8 \%$ & $15,3 \%$ & $0,9 \%$ \\
\hline Brasil & 171,98 & 353,78 & $19,8 \%$ & $12,5 \%$ & $16,1 \%$ & $6,5 \%$ \\
\hline
\end{tabular}

Fonte: Secretaria do Tesouro Naciona ${ }^{10}$ e IBGE 9 .

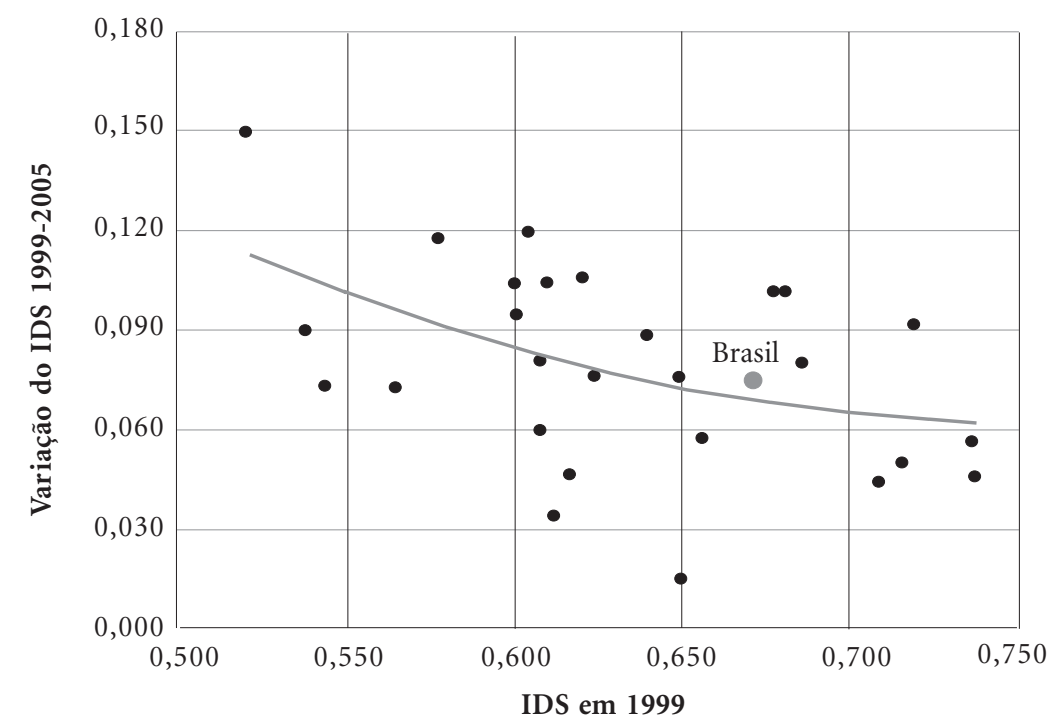

Figura 3. IDS e despesas per capita em saúde e saneamento (R\$), 2005.

Fonte dos dados originais: $\mathrm{IBGE}^{9} \mathrm{e} \mathrm{STN}^{10}$. 
que Distrito Federal, São Paulo e Rio de Janeiro ocupam posições no canto superior direito, onde maiores investimentos estão lado a lado com maiores IDS. Do outro lado, no canto esquerdo inferior, estão os estados de Maranhão, Alagoas e Pará, em posição desfavorável, pois apesar dos gastos com saúde terem crescidos, isto não foi suficiente para garantir o desenvolvimento da saúde adequado.

\section{Discussão}

Com o IDS, que agregou três dimensões da saúde, foi possível configurar o nível da saúde no Brasil em 2005 e também a progressão desse nível desde 1999. Apesar de numericamente pequeno, o conjunto de indicadores de saúde incluídos no IDS representa fatores de impacto na saúde da população brasileira e compreende os princípios do SUS.

A despeito das limitações do uso de dados agregados, que descrevem tendências gerais, vale destacar que a comparação do IDS e suas dimensões, entre as UF no Brasil, possibilitou o mapeamento das regiões com maior e menor desenvolvimento da saúde.

Em 2005, o Brasil apresentava níveis intermediários de desenvolvimento da saúde, o que representa progresso em relação aos patamares de 1999. No entanto, o panorama visualizado foi de contrastes e desigualdades. Por um lado, destaca-se um grupo de oito unidades da Federação - sete estados das regiões Sul/Sudeste e o Distrito Federal - com níveis altos de desenvolvimento da saúde, caracterizados com a melhor infraestrutura de recursos de saúde (ambulatórios, leitos hospitalares, profissionais de saúde) para prestação de assistência especializada e de maior complexidade. Essas unidades também dispõem de maior cobertura de Programas de Atenção Básica e os indicadores de saneamento (água potável e esgoto) são maiores. É também maior a eficácia de políticas públicas de saúde para a redução da mortalidade (infantil e por doenças infecto-parasitárias), da desnutrição (crianças $\leq$ 1 ano) e do baixo peso ao nascer.

Do outro lado, restaram as demais UF com IDS médio e baixo, déficits nos recursos de saúde e na cobertura dos Programas de Atenção Básica e do saneamento. Como se sabe, essas carências são históricas e já aparecem no IDS de 1999. As deficiências foram mais acentuadas em doze estados das regiões Norte/Nordeste e Centro-Oeste, configurando o panorama de disparidades regionais clássico no Brasil. Esse fato indica a necessidades de priorizar políticas públicas que superem essas diferenças e garantam o princípio da universalidade da assistência à saúde do SUS.

A deficiência de recursos de saúde apontada é outro dado preocupante, pois demonstra que na maioria das UF não há recursos para financiar uma infraestrutura de saúde adequada à prestação de assistência mais complexa e especializada e com profissionais de saúde qualificados. Nas últimas décadas, a demanda por profissionais com conhecimento e habilidades especializados vem crescendo. $\mathrm{Na}$ área da saúde, essas competências são cada vez mais desejadas não só porque novas tecnologias têm aumentado a complexidade da assistência, mas também porque o novo modelo da atenção básica, fundamentado no PSF, requer profissionais capacitados para planejar, tomar decisões e atuar, de maneira resolutiva e criativa ${ }^{11}$.

Disso decorre a importante reflexão de que as políticas públicas precisam estar voltadas não somente para incrementar quantitativamente a infraestrutura da saúde, mas também para promover a qualificação de profissionais de saúde para o desenvolvimento de competências alinhadas ao modelo de atenção básica. Isso implica rever o processo de formação acadêmica do profissional de saúde, definindo o perfil profissional mais adequado nos currículos de graduação ${ }^{12}$.

A constatação de que a cobertura dos Programas de Atenção Básica não era universal em muitas UF pode ser explicada por dificuldades na implantação destes Programas. Criado em 1994 pelo Ministério da Saúde, inicialmente o PSF teve maior inserção em municípios pequenos e foi se expandindo lentamente devido à significativa dificuldade de modificar o modelo assistencial vigente, centrado na assistência e na demanda espontânea ${ }^{11}$. Dentre outras dificuldades, relatam-se a necessidade da reestruturação da rede de serviços de saúde já existente, e da requalificação dos profissionais de saúde; a baixa adesão de profissionais de saúde e de usuários da rede de atenção básica ${ }^{7,12}$.

O baixo nível do índice de cobertura também aponta condições de saneamento deficientes. A relação positiva observada entre IDS e gastos com saúde/saneamento reforça a noção de que investimentos públicos nessa área têm impacto positivo considerável na saúde, também apontado em outros estudos. Em estudo sobre o valor futuro do IDH municipal para os municípios do Paraná, foi evidenciada uma estreita relação entre esses gastos e o Desenvolvimento Humano ${ }^{11}$. 
Por outro lado, a dimensão da eficácia mostrou-se altamente desenvolvida em todo território nacional, o que significa melhores condições de vida e sobrevida de recém-nascidos e crianças $\leq 1$ ano, além de maior capacidade da população em prevenir doenças infecto-parasitárias, o que pode ser atribuído ao crescente desenvolvimento socioeconômico e educacional do Brasil, observado nas últimas décadas. Ainda assim, foram evidenciadas desigualdades regionais na dimensão de eficácia, ficando a maioria dos estados do Norte/Nordeste em níveis inferiores aos do Sul/ Sudeste.

Essas disparidades marcantes também foram apontadas nos estudos de Souza e Filho ${ }^{14}$ e Boing e Boing ${ }^{15}$, que utilizaram a taxa de mortali- dade infantil como proxy do estado de saúde da população brasileira. A despeito das diferenças metodológicas, esses estudos demonstraram uma estreita relação entre saúde e despesa total com saúde por habitante.

Com a proposição e a análise do IDS imagina-se ter sido dado um passo metodológico no sentido de suprir a carência de indicadores sintéticos que mensurem a qualidade da saúde no país e orientem as políticas públicas. Espera-se que esse indicador auxilie os gestores da saúde no enorme desafio de criar políticas públicas coerentes com a realidade, capazes de reduzir as desigualdades, aprimorar quanti-qualitativamente a infraestrutura de saúde e tornar a saúde pública universal.

\section{Colaboradores}

MM Brevidelli trabalhou no delineamento do estudo, análise e interpretação dos dados e redação do artigo; e FCG de Freitas no delineamento do estudo, na análise e interpretação dos dados, na revisão crítica e na aprovação da versão final do artigo. 


\section{Referências}

1. Pereira MG. Principais indicadores de saúde. In: Pereira MG. Epidemiologia: teoria e prática. Rio de Janeiro: Guanabara-Koogan; 2003. p.54-61.

2. Organização Pan-americana de Saúde (OPAS). Rede Interacional de Informações para a Saúde. In: Organização Pan-americana de Saúde (OPAS) Indicadores básicos de saúde no Brasil: conceitos e aplicações. Rede Interacional de Informações para a Saúde - Ripsa. Brasília: OPAS; 2002.

3. United States Department of Health and Human Service. Health People 2010: understanding and improving health. $2^{\text {nd }}$ edition. Washington, D.C. Government Printing Office, November 2000. [site na Internet]. [acessado $2006 \mathrm{fev} 2$ ]. Available from: http://www.healthypeople.gov/Document/pdf/uih/ 2010uih.pdf

4. Fielding J, Kumanyka S. Recommendations for the concepts and form of Healthy People 2020. Am J Prev Med 2009; 37(3):255-257.

5. Programa das Nações Unidas para o Desenvolvimento (PNUD). Atlas do desenvolvimento humano do Brasil. PNUD; 2003. [site na Internet]. [acessado 2006 fev 24]. Disponível em: http://www.pnud. org.br/atlas/

6. Garcia F, Souza RC de, Castelo AM. Desenvolvimento habitacional na América Latina. In: Dowbor L, Kilsztajn S, organizadores. Economia social no Brasil. São Paulo: SENAC; 2001.

7. Brasil. Ministério da Saúde, Fundação Oswaldo Cruz. Saúde da família. Avaliação da implementação em dez grandes centros urbanos: síntese dos principais resultados. Série C Projetos, Programas e Relatórios. $2^{\circ}$ Edição atualizada. Brasília: Editora do Ministério da Saúde; 2005.

8. Brasil. Ministério da Saúde. Departamento de Informática do SUS. DATASUS. Indicadores e dados básicos 1999-2005. [internet]. [acessado $2006 \mathrm{mar}$ 7]. Disponível em: http://www2.datasus.gov.br/ DATASUS/index.php?area $=0201$

9. Instituto Brasileiro de Geografia e Estatística (IBGE). Pesquisa Nacional por Amostras de Domicílio (PNAD). 1999 a 2005. Rio de Janeiro: IBGE; 2006.
10. Brasil. Secretaria do Tesouro Nacional. Sistema Integrado de Administração Financeira do Governo Federal. [internet]. [acessado 2006 mar 7]. Disponível em: http://www.tesouro.fazenda.gov.br/estatistica/ est_estados.asp

11. Caetano R, Dain S. O Programa de Saúde da Família e a reestruturação da atenção básica à saúde nos grandes centros urbanos. Physis 2002; 12(1):11-21.

12. Campos FE de, Aguiar RAT de, Oliveira VB de. O desafio do Programa de Saúde da Família nas grandes capitais brasileiras. Physis 2002; 12(1):47-58.

13. Scarpin JE, Slomski V. Estudos dos fatores condicionantes do índice de desenvolvimento humano nos municípios do estado do Paraná: instrumento de controladoria para tomada de decisões na gestão governamental. RAP 2007; 14(5):909-933.

14. Souza TRV, Filho PAML. Análise dos dados em painel do status de saúde no Nordeste brasileiro. Rev Saude Publica 2008; 42(5):796-804.

15. Boing AF, Boing AC. Mortalidade infantil por causas evitáveis no Brasil: um estudo ecológico no período de 2000 e 2002. Cad Saude Publica 2008; 24(2): $447-455$.

Artigo apresentado em 01/06/2011

Aprovado em 28/07/2011

Versão final apresentada em 05/08/2011 